\title{
„Predigen, gefaßt in Lebenssprache“: Zur narrativen Strategie von Gotthelfs „Neuem Berner-Kalender“
}

Als die Bernische gemeinnützige Gesellschaft Ende 1838 ihrem Mitglied Albert Bitzius alias Jeremias Gotthelf die Redaktion des von ihr initierten Neuen Berner-Kalenders anträgt, nimmt der publizistisch und literarisch bereits in Erscheinung getretene Emmentaler Theologe das Angebot mit Begeisterung an. ${ }^{1}$ In einem Brief an Carl Bitzius, dem er bereits erste "Kalender-

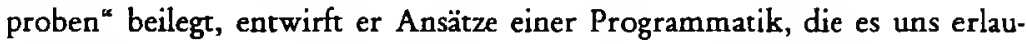
ben, die Intention, die er als Kalendermacher zu verfolgen gedachte, präziser zu beschreiben:

Es gibt einen eigenen Kalender, keinen zusammengetragenen aus Naturgeschichten und andern gemeinnützigen Langeweilbehältern. Aus Rezepten, wie Wanzen zu vertreiben seien, und wie viel Junge die Steinböckin habe, macht man keinen vernünfrigen $\mathrm{K}$ alender. Das kömmt aus der verflucht dummen gemeinnützigen Zeit, wo man im Eraste des Lebens nicht tiefer kam als zu Rezepten und in der jetzt noch unsere Staarsmänoer taumeln. Ich möchte in den Kalender Predigen bringen, d.h. hohe Wahrheiten, aber entkleidet von allem Kirchlichen, gefaßt in Lebenssprache, wie man sie auf der Kanzel nicht duldet. ${ }^{2}$

Auch an anderer Stelle hat Gotthelf sich zu den seine Kalendertätigkeit bestimmenden Zielsetzungen geäußert. Nicht eine Zusammenstellung von "Nützliche[m]" wie der "Theepflanzung" oder der "Naturgeschichte des Leoparden ${ }^{\alpha}$ habe der Volkskalender zu sein, sondern ein „Lebensbuch [...], ein Spiegel des Lebens, aber sowohl des innern als des äußern Lebens“, schreibt er 1840 an Joseph Burkhalter. ${ }^{3}$ Mit einer Metapher, die sich fast wörtlich auch bei Johann Peter Hebel findet, ${ }^{4}$ umreißt er die Funktion einer Publikationsform, die ihm in besonderem Maße geeignet scheint, eine breite, nur partiell gebildete Leserschaft anzusprechen.

Der Originalitätsanspruch, den Gotthelf in seiner Korrespondenz für die von ihm redigierten Jahrgänge des Organs der Bernischen gemeinnützigen

1 Gotthelfs andauerndes Interesse am Volkskalender äußert sich nicht zuletzt darin, daß er, wie er in seinem Brief vom 16. 12. 1838 an Carl Bitzius schreibr, bereirs früher erfolglos versucht hatte, als Kalenderautor Fuß zu fassen. Vgl. EB 4, S. 282.

2 EB 4, S. 281-282.

3 EB 5, S. 89.

4 Vgl. Hebel: Der Rheinländicche Hausfreund, S. 28 (,ein wohlgezogener Kalender soll seyn ein Spiegel der Welt \%). 
Gesellschaft erhebt, wirft die Frage nach dem Verhältnis zwischen dem Neuen Berner-Kalender und anderen Volkskalendern auf: Vom publizistischen Konzept, das den früheren Jahrgängen des Neuen Berner-Kalenders zugrunde lag, grenzt Gotthelf sich in aller Deutlichkeit ab. Der wenig erfolgreiche Neue Berner-Kalender für das Jabr 1838 enthält neben dem durch Rätsel, Anekdoten, Aphorismen, Scharaden und kurzen Gedichten aufgelockerten Kalendarium eine Reihe naturkundlicher, historischer und landwirtschaftlicher Informationen sowie Beiträge, die der sittlichen Belehrung der Landbevölkerung dienen sollen, und bietet damit im wesentlichen jene Kompilation volksaufklärerischer Instruktionen, die Gotthelf für die von ihm zu verantwortenden Kalenderjahrgänge explizit ablehnt. Trotzdem sind die in einer Reihe von Briefen zum Ausdruck gelangenden programmatischen Positionen nicht singulär. In der Ablehnung eines Kalenders, der sich auf gemeinnützige Indoktrination beschränkt, geht er einig mit Gottlieb Jakob Kuhn, dem Herausgeber des traditionsreichen und weit verbreiteten Berner Hinkenden Boten. Mit Skepsis hatte dieser die Bestrebungen der Bernischen gemeinnützigen Gesellschaft, ein eigenes Kalenderprojekt zu initiieren, begleitet und insbesondere auf die Problematik einer allzu sehr volksaufklärerischer Didaxe verpflichteten Schreibart hingewiesen. ${ }^{5}$ Das Ergebnis der publizistischen Bemühungen der "Alleinweisen ${ }^{\alpha 6}$, wie er die Vorstandsmitglieder der Bernischen gemeinnützigen Gesellschaft ironisch bezeichnete, vermochte ihn nicht zu befriedigen. Der Kalender sei nicht nach dem "Geschmacke [des] Volkes“, habe ${ }_{n}$ zu viel Schulmeisterliches, Trockenes, zu wenig Heiterkeit und Salz. ${ }^{\alpha 7}$ Zwar spricht sich der erfahrene Kalendermacher Kuhn nicht grundsätzlich gegen das „Kluge ${ }^{\alpha}$ und das „Nützliche ${ }^{\alpha}$ aus, entscheidend sei jedoch, daß ein an breitere Publikumsschichten gerichtetes Periodikum den „Volkston nicht verliere ${ }^{\alpha 8}$, denn, ,[w]enn man will, daß der Kalender unter dem Volke kursiere und Interesse finde, so muß man mit dem Volke seine Sprache reden. ${ }^{\text {"9 }}$ Schon Johann Peter Hebel hatte 1806 in seinem Unaufgeforderten Gutachten über eine vortheilhaftere Einrichtung des Calenders betont, "wie viel weiser es sey, den Geschmack seines Publikums zu benutzen, als zu verachten und beleidigen. ${ }^{.10}$ Als Verfasser eines derart an den Lesegewohnheiten der Rezipienten orientierten Kalenders schwebte ihm ein Kalendermann vor, der "beobachtend mit und unter dem Volke

${ }^{5} \mathrm{Zu}$ Kuhns diesbezüglichen Äußerungen vgl. Hunziker: Der Newe Berner-Kalender, S. $101-108$.

${ }^{6}$ Zitiert nach Hunziker: Der Neue Berner-Kalender, S. 102.

7 Ebenda S. 107.

8 Ebenda S. 102.

9 Ebenda S. 104.

${ }^{10}$ Das Gutachten ist abgedruckt in Rohner: Kommentarband zum Rheinländischen Haus. freund, S. 75-79, hier S. 78. Zu Hebels Unaufgefordertem Gutachten vgl. Voit: Kalenderwesen, S. 93-115. 
lebt ${ }^{\alpha,}{ }^{11}$ der die Welt seiner Adressaten aus eigener Erfahrung kennt. Auch Gotthelf fordert vom Kalenderautor, daß ner das Leben, welches er beschreiben will, kennen [müsse] aus eigener Anschauung “, ${ }^{12}$ daß er "das Volk und seine Not, sein wahr Wohl, seine Vergangenheit, seine Gegenwart und seine Zukunft" im Auge haben solle. ${ }^{13}$

In der Ablehnung einer ausschließlich gemeinnütziger Belehrung verpflichteten Schreibart, im Bestreben, den Lesebedürfnissen und -gewohnheiten breiterer Bevölkerungsschichten Rechnung zu tragen, geht Gotthelf mit Kuhn und Hebel einig. Die programmatische Nähe zu anderen Herausgebern populärer Kalender sollte jedoch nicht den Blick verstellen für die signifikanten Akzentverschiebungen, die der Berner Dichter im Zuge der Konzeptualisierung seines Neuen Berner-Kalenders vornimmt. Der rekurrierende Hinweis auf die "hohen Wahrheiten “, die der Kalender vermitteln soll, ${ }^{14}$ die Darstellung des Volksschriftstellers als strenge, von "heiliger Liebe" getriebenen Vaterfigur, die, ganz im Sinne des Bibelworts, das Sohnesliebe und Züchtigung kausal verknüpft (vgl. Sprüche 13, 24), die Leserschaft "geißelt nicht aus Bosheit, sondern aus innigem Erbarmen ", ${ }^{15}$ offenbart die theologisch-moralische Intention Gotthelfs. Die Betonung der „Freimütigkeit $^{\alpha 16}$ wiederum zielt auf die politische Ausrichtung seiner schriftstellerischen Tätigkeit. Freimütigkeit bedeutet in diesem Zusammenhang die Bereitschaft, auch die Obrigkeit einer kritischen Beurteilung zu unterziehen, denn:

Das Volk ärgert sich an nichts mehr, als wenn es immer und immer als einzig zu heilendes Glied dargestellt wird, die Repräsentanten der andern Stände aber ihm zum Muster gegeben werden als Tugendbilder. Das Volk fühlt dieses Unrecht scharf und verliert das Zutrauen zum Buch und zum Schriftsteller. ${ }^{.7}$

Als Adressaten von Kalenderliteratur geraten damit auch die Repräsentanten staatlicher Strukturen ins Blickfeld. Der Verfasser populärer Schriften, wie ihn Gotthelf idealtypisch definiert, wendet sich nicht primär an eine Masse Unmündiger, die es zu belehren gilt, sondern antwortet schreibend auf die politische, soziale, ökonomische Wirklichkeit seiner Zeit. Wenn der Emmentaler Pfarrer an anderer Stelle Volkstümlichkeit und Republikanis-

\footnotetext{
11 Rohner: Kommentarband zum Rheinländischen Hausfreund, S. 78.

12 EB 5, S. 333. Zu Gotthelfs Konzeption des Volksschriftstellers vgl. Holl: Gotthelf im Zeitgeflecht, S. 21-32.

13 EB 5, S. 248.

14 Neben der bereits zitierten Stelle aus einem Brief an Carl Bitzius (EB 4, S. 281-282) vgl. insbesondere den Brief an Irenäus Gersdorf vom 28.9.1843 (EB 5, S. 331-336, hier 333f.)

is EB 5, S. 334.

${ }^{16}$ EB 5, S. 334.

17 EB 5, S. 334.
} 
mus verknüpft, ${ }^{18}$ unterstreicht er noch einmal die Notwendigkeit von populären Lesestoffen, die sich ihre Rezipienten nicht als unberatene Untertanen, sondern als autonome Bürger denken.

Gotthelfs knappe und eher unsystematische briefliche Äußerungen zu seiner Tätigkeit als Kalenderautor erscheinen merkwürdig ambivalent: Sie knüpfen einerseits an verbreitete Beschreibungsmuster volkstümlichen Dichtens an und fordern andererseits von einem gelungenen Kalender die durchaus neuartige Kombination von Paränese und Zeitkritik. Ungeachtet der Tatsache, daß bauernaufklärerischen Bestrebungen eine Absage erteilt wird, ${ }^{19}$ daß eine Haltung, die die Rezipienten zu Objekten gemeinnütziger Indoktrination degradiert, abgelehnt und die religiöse und politische Mündigkeit breiterer Publikumsschichten postuliert wird, erscheint der Kalendermacher in der Rolle eines Verkünders zeitlich und überzeitlich gültiger Wahrheiten und kann damit eine Autorität in Anspruch nehmen, die die Vorstellung einer autonomen Perzeption des Kalenders durch den Leser relativiert. Unter partieller Berücksichtigung des in anderen Volkskalendern modellhaft Präfigurierten gestaltet Gotthelf einen Kalender, der sich in mehrfacher Hinsicht von traditionellen Vorgaben entfernt und in seiner Eigenwilligkeit nicht nur bei Gotthelfs Zeitgenossen zu Irritationen geführt hat.

1.

Was die Spezifität an Gotthelfs Kalender ausmacht, ist erst auf den zweiten Blick zu erkennen. ${ }^{20}$ Der erste vom Emmentaler Pfarrer eigenverantwortlich verfaßte und edierte Jahrgang, der Neue Berner-Kalender für das Jabr 1840, ${ }^{21}$ unterscheidet sich äußerlich nicht wesentlich von seinem Vorgänger. Aufmachung und Titelblatt entsprechen den Ausgaben von 1838 und 1839, das

${ }^{18}$ EB 6, S. 246.

${ }^{19}$ Gotthelfs Tätigkeit als Kalenderschreiber ist trotz der Abgrenzung von zeitgenössischen gemeinnützigen Bestrebungen im Kontext der Volksaufklärung zu sehen. Die Aufhebung der Trennung von Ökonomie und Sittenerziehung sowie die Integration religiöser Unterweisung in volksaufklärerischem Schriftum hat Holger Böning als Kennzeirhen der volksaufklärerischen Bemühungen insbesondere des 19. Jahrhunderts hervorgehoben (vgl. Böning: Genese der Volksaufklärung, S. XIVII). Die ambivalente Haltung Jeremias Gotthelfs der Volksaufklärung gegenüber dokumentiert am Beispiel ausgewählter Romane Braungart: Aufklärungskritische Volksauflkïrung.

${ }^{20} \mathrm{Zu}$ Gotthelfs Neuem Berner-Kalender gibt es nur wenig Forschungsliteratur. Abgesehen von Walter Muschgs Vorwort zu dern von ihm herausgegebenen Schatzkästlein des Newen Bemer Kalenders (Gorthelf: Scharzkjötlein des Neuen Berner Kalenders, S. 5-32) sind Hans Itrens eher problematische Dissertation (Itten: Gotthelf als Kalenderschreiber) sowie Rohner: Kalendergeschichte und Kalender, S. 327-339 zu nennen.

${ }^{21}$ Gotthelfs Beiträge für den Newen Berner Kalender sind abgedruckt in SW 23 und 24. Walter Muschg hat eine Reihe von Gotthelfs Kalendergeschichten herausgegeben, siehe Anm. 20. 
Kalendarium wurde, leicht modifiziert, übernommen. Unterschiede ergeben sich primär hinsichtlich des Erzählteils, der unter Gotthelfs Redaktion beträchtlich an Umfang gewinnt. Bemerkenswert ist nun, daß der Neue Berner. Kalender für das Jabr 1840 zunächst in etwa das zu enthalten scheint, was der Leser auch von anderen zeitgenössischen Kalendern erwarten durfe. Berichterstattung über aktuelle Begebenheiten (Einiges aus dem eidgenössischen Lustlager zu Sursee, Die scbōnen Käsereien und die wüsten Männer, Das Krokodil), nützliche Rezepte (Gemeinnütziges), eine Genreszene (Brucbstück aus der Berner Welt), moralisches Exemplum (Bedenkliche Verlegenbeit), Schwank (Wer lügt am Besten) und Anekdoten (Mißverständnisse, Fatale Zumutbung, Naive Vorstellungen, Genügsamkeit, Wer paßt gut zusammen) gehören zum Inventar volkstümlicher Kalender. Bei näherem Hinsehen erweist sich die Zuordnung der Erzähltexte zu den obengenannten poetologischen Kategorien allerdings als äußerst problematisch, denn jeder Text wird, unabhängig vom Stoff, den er gestaltet, zum Träger Gotthelfscher Zeitkritik: Die Berichterstattung vermittelt nicht sachliche Information, sondern dient einer satirischen Abrechnung mit politischen, ökonomischen und sozialen Gegebenheiten, die Rezepte ironisieren die Leichtgläubigkeit der Leser, die Genreszene entpuppt sich als scharfe Kritik am Dünkel städtischer Dienstboten, die Anekdoten nehmen verbreitete Ansichten aufs Korn. Gotthelf greift auf seinem Publikum vertraute Stoffe und Motive zurück, um sie ironisch zu unterlaufen; was bei andern Kalendermachern didaktischen Zwecken dient, wird bei Gotthelf zum Medium einer Polemik, die eine Vielzahl von Lebensbereichen umfaßt.

Neben der satirischen findet sich im Neuen Berner-Kalender für das Jabr 1840 als weitere dominante Schreibart die geistliche Ermahnung. Sie klingt in einigen der bereits genannten Beiträge an und kennzeichnet insbesondere diejenigen Texte, die auch in stofflicher Hinsicht eine Abgrenzung zwischen Gotthelfs Publikation und anderen Volkskalendern erlauben. Es handelt sich zum einen um die Paränesen Der Glaube, Seltsamer Trost und Das Wetter sowie um die legendenhatten Erzählungen Der bekebrte Mordiofubrmann, Der russische Knabe, Das gelbe Vögelein und das arme Margrithli und Die beiden Raben und der Holzschelm. Der Predigtzyklus über die Kardinaltugenden, zu dem im Neuen Berner-Kalender für das Jabr 1840 die Homilie Der Glaube den Auftakt bildete, findet mit Die Hoffnung (1841), Die Liebe (1842), Die Furcht (1843), Demut (1844) und Die Sanftmut (1845) seine Fortsetzung. Paränetischen Charakter haben auch eine Reihe von in den späteren Kalenderjahrgängen abgedruckten Beiträgen, die Sünden wie Geiz, Liederlichkeit oder Hochmut anprangern.

Das den programmatischen Äußerungen zu entnehmende Postulat einer Verbindung von Sozialkritik und religiöser Erbauung hat Gotthelf, wie ein erster Blick in die von ihm redigierten Ausgaben des Neuen Berner-Kalenders zeigt, literarisch umgesetzt. Die politische, ökonomische und soziale Themen 
behandelnden Beiträge werden ergänzt durch theologische Betrachtungen, die Gotthelfs Publikation in die Nähe der religiösen Volkskalender rücken. ${ }^{22}$ Satire und Paränese bilden - und dies gilt für die zwischen 1840 und 1845 erschienenen Jahrgänge des Neuen Berner-Kalenders in zunehmendem Maße - die den Intentionen Gotthelfs gemäßen Darstellungsmodi menschlicher Erfahrung. Enthalten die frühen Jahrgänge noch Konzessionen an den Geschmack des Publikums, werden die späteren Ausgaben immer mehr zum Medium der spezifischen Anliegen ihres Verfassers. Die erzählenden und primär unterhaltenden Beiträge treten immer mehr in den Hintergrund, eine signifikante Reduktion der Themenvielfalt zeichnet sich ab, die Beiträge gewinnen einen Umfang, der das für Kalender übliche Maß sprengt. Obwohl Gotthelf sich vor allem in den ersten beiden der von ihm betreuten Jahrgänge um eine thematische Gliederung bemüht und auf unterschiedliche Gattungen rekurriert, wirkt der Neue Berner-Kalender zunehmend gleichförmig, erscheint schließlich als bemerkenswert homogenes Periodikum voll innertextlicher Bezüge, das völlig im Dienste seines Autors steht. Symptomatisch für die hier beschriebene Entwicklung ist der neue Untertitel, mit dem Gotthelf ab 1843 seine Publikation versieht. Nachdem der Neue BernerKalender während mehrerer Jahre als „Ein nützliches Handbuch zur Unterhaltung und Belehrung" erschienen war, stellt er sich nun seinen Lesern als „Ein Buch für die Gewissen, welche gutmüthige Wahrhafrigkeit ertragen können" vor.

An den sein Wirken als Kalenderautor bestimmenden Intentionen hält Gotthelf in all den von ihm zu verantwortenden Jahrgängen fest, und dies ungeachret der Modifikationen, denen er seinen Kalender bis 1845, dem $\mathrm{Jahr}$, in dem der sechste und letzte von ihm betreute Jahrgang erscheint ${ }^{23}$, unterzieht. Die konzeptuellen Verschiebungen, die Gotthelf zwischen 1840 und 1845 vornimmt, lassen die bereits für den ersten von ihm verfaßten Neuen Berner-Kalender charakteristischen Tendenzen noch deutlicher zutage

21 Ursula Brunold-Bigler rechnet den Neuen Berner-Kalender ohne weitere Begründung zu den religiösen Volkskalendern (vgl. Brunold-Bigler: Die religiösen Volkskalender in der Schweiz im 19. Jahrhundert, S. 55-56). Für Gotthelfs Affinität zum religiösen Volkskalender dürfte sprechen, daß der Berner Theologe nach 1845 Beiträge für in Deutschland erscheinende konfessionelle Kalender beigesteuert hat (vgl. Bauer: Geistliche Restauration der Biedermeierzeit, S. 149).

23 Über die Ursachen fiir die abrupte Beendigung von Gotthelfs Kalendertätigkeit ist gemutmaßt worden. Der Widerstand, auf den der Newe Berner-Kalender bei den Behörden und der liberalen Presse stieß (vgl. Hunziker: Der Neue Bemer Kalender, S. 143), politische Konflikte, die in Gotthelfs Absetzung als kantonaler Schulkommissär kulminierten (Muschg [Hrsg.]: Gotthelf. Schatzkästlein des Newen Berner Kalenders, S. 11) wurden als mögliche Gründe genannt. Entscheidender scheint zu sein, daß es zwischen Gotthelf und seinem Verleger Christian Albrecht Jenni zum Zerwürfnis gekommen war. Im Brief vom 8.1.1846 an Irenäus Gersdorf schreibt er: „Für dieses Jahr habe ich keinen [Kalender] herausgegeben; mein Buchhändler war radikal geworden, da habe ich ihm aufgekündet und start seiner keinen andern gesucht ${ }^{\alpha}$ (EB 6, S. 248). 
treten: Eine erste signifikante Änderung betrifft den Bereich des, Gemeinnützigen'. Schon 1841 fallen die Anleitungen zum Schröpfen und Purgieren weg, ab 1842 verzichtet Gotthelf sowohl auf die Aderlaßtafel als auch auf die die bäuerliche Haushaltsführung betreffenden Ratschläge. Gleichzeitig gewinnen Beiträge, die sich kritisch mit Aberglauben und Quacksalberei auseinandersetzen, an Gewicht: Mit satirischer Schärfe geißeln Einige Hausmittelchen (1841), Eigene Meinungen (1843), Medizinisches (1844) und Heilsame Dreck-Apotbeke (1845) die Auswirkungen medizinischer Scharlatanerie. Gotthelfs Polemik gegen die Auswüchse der Volksmedizin hängt nicht zuletzt damit zusammen, daß 1842 die bernische Sanitärskommission an ihn gelangt war, mit der Bitte, er möge sich publizistisch an den durch die Behörden in Gang gesetzten Bestrebungen zur gesundheitlichen Aufklärung der Bevölkerung beteiligen. Die Kommission wünschte insbesondere zu erfahren, ob der Pfarrer von Lützelflüh

im Falle wärę[n], über den fraglichen Gegenstand [...] eine für das Volk bestimmte Schrift zu verfassen, oder ob [er] nicht vielleicht zweckmäßiger erachten würde[n], in dem von [ihm] redigierten Berner-Kalender in beliebigen Abschnitten alljährlich diese Materie in der [ihm] als bewährtem Volksschriftsteller eigenen ansprechenden Schreibart zu behandeln. ${ }^{24}$

Gotthelf hat die Anregung aufgegriffen; seiner Zusammenarbeit mit der Sanitätskommission sind nicht nur der Roman Anne Bäbi Jowäger, sondern auch die obengenannten Kalenderbeiträge zu verdanken.

Als neue Rubrik werden 1841 die Kuriositäten eingeführt. Sie knüpfen an die in andern Kalendern verbreiteten und beim Publikum beliebten, Weltbegebenheiten ${ }^{25}$ an und schildern in chronologischer Reihenfolge Ereignisse, die sich im jeweils vorangegangenen Jahr zugetragen haben. Die Bezeichnung ,Kuriositäten' verweist auf die satirische Tendenz von Gotthelfs sehr subjektiv gefärbter Kalenderchronik. Was dem Leser geboten wird, ist nicht historische Berichterstattung, sondern eine eigenwillige Auswahl an Begebenheiten, die dem Autor als Ausgangpunkt für die polemische Abrechnung mit den politischen Verhältnissen dienen. Ebenfalls 1841 erscheint mit Die Schelmenzucht der erste Teil der Reisebilder aus den Weltfabrten eines Scbneiders, die Gotthelf bis 1845 jährlich fortsetzt. Mit den Kuriositäten verbindet die Reisebilder aus den Welffabrten eines Schneiders die Kritik am bernischen Staat, am Rechtswesen, an den zentralistischen Bestrebungen der Eidgenossenschaft. Das Interesse an aktuellen politischen und sozialen Problemen und Diskursen manifestiert sich in einer ganzen Reihe von Beiträgen, in denen der Bogen von den Folgen der Industrialisierung (Marei, die Kuderspinnerin, und

24 EB 5, S. 193.

${ }^{25}$ Die Bezeichnung, Weltbegebenheiten' verwendet Johann Peter Hebei mehrfach in seinem Rheinländischen Hausfreund (vgl. Hebel: Der Rheinländische Hausfreund, S. 71, 75, 236, 240, 254). 
ibr Tröster 1841), über die Emanzipation der weiblichen Dienstboten (Der große Kongreß auf dem Kasinoplatz in Bern 1842) bis hin zur Jesuitenfrage (Die Jesuiten und ibre Mission im Kanton Luzern 1844) gespannt wird.

Gemeinsam ist den in kritischer und in erbaulicher Absicht verfaßten $\mathrm{Ka}$ lenderbeiträgen der allegorische Charakter der narratio. Das erzählte $\mathrm{Ge}$ schehen erscheint als Sinnbild menschlicher Möglichkeiten und Grenzen und als Veranschaulichung göttlichen Wirkens in der Welt. Das Interesse richtet sich weniger auf die Historizität des Dargestellten als vielmehr auf dessen Verweischarakter. Nicht das geschichtliche Faktum interessiert - die sich auf vergangene oder aktuelle Ereignisse beziehenden Beiträge bleiben in der Regel bezüglich Datierung und Situierung fragmentarisch, vermischen historisch Verbürgtes und Fiktion - sondern dessen Bedeutung im Rahmen einer Welt, die dem ordnenden Zugriff Gottes unterliegt. Satire und Paränese erweisen sich als letztlich funktionsäquivalent: Durch die Demaskierung des Bösen und die Ermunterung zum Guten soll ein christliches Verhaltensideal propagiert werden, das noch der Verwirklichung harrt. Folgerichtig hat das delectare hinter das prodesse zurückzutreten, denn:

Der Kalender ist kein Betbuch, aber er ist ein Buch, das zur Besserung der Menschen beitragen soll wie jedes andere Buch; und wenn ein Buch das nicht will, so verdient es, verboten zu werden. Aber der Unterschied zwischen dem Kalender und einem Betbuch ist, daß das, was das Betbuch zur Bekehrung des Menschen auf eine geistliche Art sagt, der Kalender auf weltliche Art darstellt. ${ }^{26}$

2.

Auf welche Weise nun versucht der Kalenderautor Gotthelf eine letztlich religiöse Botschaft in "weltlichen“"Worten zu vermitteln? Um eine Antwort auf diese Frage zu finden, sollen im folgenden die für den Neuen Ber. ner-Kalender konstitutiven Kommunikationsmuster einer Analyse unterzogen werden. Daß der Kalender in besonderem Maße geeignet sei, einen Dialog zwischen Autor und Lesern in Gang zu setzen, ist ein topos sowohl der Kalenderliteratur als auch der Kalenderforschung. ${ }^{27}$ Bereits in den frühneuzeitlichen Kalendern gewinnt ein Kalendermann Gestalt, der mit seinen Lesern das Gespräch aufnimmt. Als Hausfreund bei Hebel, als Hinkender Bote bei Kuhn gelangt der Kalendermann gar aufs Titelblatt und illustriert so die Bedeutung, die ihm als Personifikation kommunikativer Kompetenz zukommt. Auch Gotthelf bedient sich der Figur des Kalendermannes, um den dialogischen Charakter seines Schreibens deutlich werden zu lassen. Schon

${ }^{26} \mathrm{SW} 23, \mathrm{~S} .13$.

${ }^{27}$ So hat beispielsweise Jan Knopf den dialogischen Charakter als primäres Merkmal des $\mathbf{K}_{2-}$ lenders bestimmt (Knopf: Kalendergeschichte, S. 23f). 
im Neuen Berner-Kalender auf das Jabr 1840 tritt er in Erscheinung, ${ }^{28}$ im darauffolgenden Jahrgang wendet er sich in den Homilien Die Hoffnung und Das Brot direkt an sein Publikum, ${ }^{29}$ erscheint in Marei, die Kuderspinnerin, und ibr Tröster sowie in den zum Zyklus Reisebilder aus den Weltfahtrten eines Schneiders gehörenden Beiträgen als in der Ich-Form berichtende Figur, ist Adressat des fiktiven Briefs, den eine Kuh, die "ehrsame Frau Kleb" an den Kalendermacher schickt. ${ }^{30}$ Die Bedeutung, die Gotthelf dem Dialog beimißt, offenbart sich nicht nur in der Dominanz der Figurenrede im Neuen Berner-Kalender, auf die schon Ludwig Rohner hingewiesen hat, ${ }^{31}$ sondern wird auch in einem Brief an Irenäus Gersdorf aus dem Jahr 1846 thematisch: Er habe gelernt zu hören, schreibt Gotthelf, „was selten Herren könn[t]en." Denn:

Entweder schaffen sie sich die Bauern so schnell als möglich vom Leibe, oder sie dozieren ihnen, geben ihnen ihre Weisheit maßweise ein, oder sie fragen direkt wie Untersuchungsrichter. Dieses alles schneidet offenen Mitteilungen jeglichen Faden ab. Man muß Gespräche einleiten können, im Gang erhalten, unbemerkt einhelfen, nie merken lassen, daß man besondere Bedeutung auf etwas lege [...], dann vernimmt man etwas und kömmt doch täglich zu neuen Dingen. ${ }^{32}$

Was Gotthelf hier entwirft, ist ein Modell zwischenmenschlicher Kommunikation, das auf reziprokem Austausch basiert, das allen beteiligten Dialogpartnern Raum läßt für Äußerungen. Der Autor erscheint nicht als derjenige, der sich mit auktorialem Gestus an seinen Leserkreis wendet, sondern als aufmerksamer Zuhörer, der für die Welt einer ländlichen Bevölkerungsschicht Interesse bekundet. $\mathrm{Zu}$ fragen ist nun allerdings, inwiefern der Neue Berner-Kalender das hier beschriebene Ideal einlöst. Die exemplarische Analyse des im Neuen Berner-Kalender für das Jabr 1840 erschienenen Beitrags Die Schelmenzucht ${ }^{33}$ dürfte zeigen, daß zwischen poetologischem Programm und literarischer Praxis eine offensichtliche Divergenz besteht.

Die satirische Erzählung, in der ein weitgereister Schneider und sein Komplize der im Wirtshaus versammelten bäuerlichen Bevölkerung die Vorteile eines Justizsystems, das den Dieben freie Hand läßt, schmackhaft zu machen versucht, ist vor dem Hintergrund der Bemühungen um eine Rechtsreform im Kanton Bern zu lesen. ${ }^{34}$ Die herrschende Rechtsunsicherheit - bis in die Mitte des 19. Jahrhunderts stützten sich die Richter bei der Urteilsfindung mangels einer verbindlichen Strafgesetzgebung auf veraltete

28 Vgl. SW 23, S. 79.

29 Vgl. SW 23, S. 119 sowie S. 143-152 passim.

30 Vgl. SW 23, S. 218-226.

31 Vgl. Rohner: Kalendergeschichte und Kalender, S. 335.

32 EB 6, S. 246.

33 SW 23, S. 195-203.

${ }^{34}$ Zur bernischen Rechtsreform und Gotthelfs diesbezüglicher Kritik vgl. Dürrenmatt: Die Kritik Gotthelfs am zeitgenössischen bernischen Recht. 
Gerichtssatzungen und das Gewohnheitsrecht - hatte die liberale Berner Regierung in den 1830er Jahren dazu bewogen, die Ausarbeitung neuer strafrechtlicher Normen in die Wege zu leiten. Als einer der mit der Erarbeitung eines neuen Gesetzes betrauten Juristen legte der deutsche Emigrant Wilhelm Snell, der an der Universität Bern einen juristischen Lehrstuhl inneharte, Richtlinien für die Behandlung von Eigentumsdelikten vor, die von Gotthelf scharf kritisiert wurden. ${ }^{35}$ Die Schelmenzucht ist nicht zuletzt der Versuch, einer von naturrechtlichen Prämissen ausgehenden Rechtsauffassung, wie sie Wilhelm Snell vertrat, die Vorstellung einer auf biblische Positionen rekurrierenden Idee von Gerechtigkeit entgegenzustellen. Dies geschieht im Rahmen einer narrativen Strategie, die nicht auf direkte Didaxe setzt, sondern auf die mit Hilfe erzählerischer Mittel bewerkstelligte Dekonstruktion der im Text dargestellten ,Schelmenzucht'.

Die Erzählung besteht aus Rahmengeschichte - ein Ich-Erzäbler begibt sich ins Wirtshaus und trifft dort nicht nur auf seine Nachbarn und Freunde, sondern auch auf einen Schneider, der von wunderbaren Erlebnissen berichtet - und zwei parallelen Binnengeschichten, in denen der Schneider,

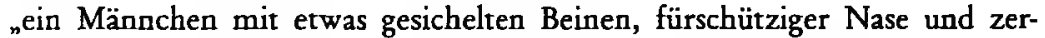
stochenem Zeigfinger ${ }^{46}$ die ,Schelmenzucht ${ }^{36}$ schildert, und ein weiterer Gast, „eine hagere Gestalt “, die einem "wandernden Baßgeiger " gleicht, ${ }^{37}$ deren Nutzen verteidigt. Gleich zu Beginn wird damit ein Kontrast konstituiert zwischen den Protagonisten des Rahmens und denjenigen der Binnenerzählungen. Handelt es sich bei ersteren um Vertreter der im Dorf verwurzelten Bevölkerung, so repräsentieren der Schneider und der Baßgeiger sozial desintegrierte Individuen, Außenstehende. Der Gegensatz zwischen den beiden Figurengruppen manifestiert sich auf vielfältige Weise. Der Seßhaftigkeit der Landbewohner steht die nomadisierende Lebensweise des Schneiders gegenüber, der von sich behauptet, „er sei die ganze Welt umreist ${ }^{4}{ }^{38}$ und des "wandernden ${ }^{*}$ Baßgeigers. Dem Kontrast $\mathrm{zwischen}$ räumlicher Statik und Dynamik entspricht das Redeverhalten der verschiedenen Handlungsträger. Anders als die Bauern, die sich, beispielsweise beim Zutrinken, ritualisierter Sprachformeln bedienen und auf eindeutige Aussagestrukturen wie ,es duech ne, e Schelm syg e Schelm, $u$ allbets heyg me se ghäycht, u de sygs o für gsi “" ${ }^{39}$ zurückgreifen, nutzen sowohl der Schneider als auch der Baßgeiger die Sprache, um konventionelle Relationen zwischen Wort und Bedeutung zu durchbrechen. Die legitime Klage eines bestohlenen Krämers wird als „groblecht ${ }^{*}$ bezeichnet, ${ }^{+0}$ aus einem Dieb, der

\footnotetext{
35 Vgl. ebenda S. 126-132.

$36 \mathrm{SW} 23$, S. 196.

37 SW 23, S. 201.

38 SW 23, S. 196.

39 SW 23, 5. 197.

$40 \mathrm{SW} \mathrm{23,} \mathrm{S.} 198$.
} 
den Vorratsspeicher eines Bauern leergeräumt hat, wird ein „Spycherfin$\operatorname{der}^{4}{ }^{41}$ Konsequent verwendet der Schneider für die Opfer der von ihm geschilderten Eigentumsdelikte negativ konnotierte Begriffe, während er das Handeln der Diebe mit positiv besetzten Termini beschreibt, um auf diese Weise eine Kriminalisierung der Opfer beziehungsweise eine Entkriminalisierung der Täter zu bewirken. Noch raffinierter verfährt der Baßgeiger, wenn er seine apologetische Rede mit einer religiösen Begrifflichkeit durchsetzt, die dazu dient, den Verstoß gegen das achte Gebot zu legitimieren. Wendungen wie "für die Seelen heilsam", „sich auf Gott verlassen", „den Mammon anbeten ", ${ }_{n}$ sich in Geduld fassen ${ }^{\text {, }}$, Demut und Trost " und ${ }_{\text {"Reich der Gerechtigkeit }}{ }^{42}$ sowie die Parodie biblischer Verse ${ }^{43}$ suggerieren eine Kongruenz mit genau demjenigen theologisch fundierten Normensystem, das der Baßgeiger in seiner Argumentation außer Kraft setzt.

Welcher rezeptionssteuernder Verfahrensweisen bedient sich nun der als Ich-Erzähler in Erscheinung tretende Kalendermann, um sicherzustellen, $\mathrm{da} B$ seine Leser die demagogischen Aussagen des Schneiders und seines Spießgesellen durchschauen? Zum einen wird die Glaubwürdigkeit des Schneiders gleich zu Beginn in Frage gestellt. Zwar beruft sich dieser auf seine Welt-Erfahrung als Garantin für den Wahrheitsgehalt seiner Erzählung; was er seinen Zuhörern allerdings berichtet, trägt deutlich fiktionale Züge: Die Hyperbolik seiner Reisebeschreibung," die Figurenkonstellation von König, Königstochter und Schneider sowie Handlungmuster wie die Heirat einer Prinzessin mit einem verdienten, jedoch sozial nicht ebenbürtigen Mann auf Wunsch des Vaters oder die wunderbare und plötzliche Wendung des Unglücks in dauerhaftes Wohlbefinden verweisen auf das Märchen. Damit ist die Wahrheit des Schneiders als fragwürdige entlarvt, was er berichter, steht von Anfang an unter dem Verdacht der Lügenhaftigkeit.

Realitärsverhafteter wirken die Exempla, die der Schneider erzählt, um die Auswirkungen der ,Schelmenzucht' zu illustrieren. Die Ereignisse spielen nun in einer Welt, die den Wirtshausgästen und den Kalenderlesern gleichermaßen vertraut ist. Die Geschädigten sind Bauern, Krämer und Handwerker, das Diebesgut besteht aus Rössern, Strümpfen, kleinen Geldsummen, Hammen, Speckseiten, Kitteln, Tuch und Käse, aus Dingen also, die jeder der Leser in kleinerer oder größerer Quantität besitzt. Der Bezug zur Wirk-

$41 \mathrm{SW} 23$, S. 199.

42 SW 23, S. $202 f$.

43 So parodiert der Satz „Denn was hilft erben und gewinnen, wean es einem hintendrein die Schelmen nehmen?“ (SW 23, S. 202) Mark. 8,36, wo es heißt: „Denn was hülfe es dem Menschen, wenn er die ganze Welt gewönne und nähme an seiner Seele Schaden?"

4 Vgl. SW 23, S. 196: „Denn er sei in Städten gewesen, wo man sieben Tag und sieben Nächte härte fahren müssen, wenn man von einem Tor zum andern gewollt, und in Ländern, wo die Sonne nicht groß genug gewesen sei, um heiter zu machen, sondern man noch nebenbei die Gasbeleuchtung hätte einführen müssen." 
lichkeit der Adressaten ermöglicht es diesen, sich als potentielle Opfer zu sehen, sich mit dem Berichteten zu identifizieren. Die Reaktion der Wirtshausgäste zeigt denn auch, daß sie die Ausführungen des Schneiders und des Baßgeigers im Hinblick auf ihre eigene Erfahrungswirklichkeit reflektieren und daraus ihre Antwort auf die "Schelmenzucht' ableiten. Aus anfänglicher Skepsis, verkörpert in der Figur eines „schwerfälligen Bauern“, ${ }^{45}$ wird offene Ablehnung, ${ }^{\text {th }}$ und am Ende schreiten die Zuhörer zur Tat, indem sie die Gaststube fluchtartig verlassen und sich damit nicht nur innerlich, sondern auch räumlich von den Darlegungen der Verfechter einer neuen Justizordnung distanzieren. Ihre Reaktion veranschaulicht auf exemplarische Weise die vom Erzähler intendierte Rezeption der Schelmenzucht durch das Kalenderpublikum. Der adäquaten Wahrnehmung der Wirtshausbesucher soll diejenige der Leser entsprechen; deren Erkenntnisprozeß wird im Text durch Identifikationsfiguren antizipiert. Die Ineinssetzung mit den Protagonisten des Erzählrahmens wird nicht nur dadurch erleichtert, daß diese sozial sowie durch den Gebrauch der Mundart - derer sie sich im Gegensatz zum Schneider und Baßgeiger bedienen - auch sprachlich den Adressaten des Neuen Berner-Kalenders angenähert werden. Auf einen identifikatorischen Zugang zum Dargestellten zielt auch der Wechsel der Redeform: hatte der Erzähler die Reiseerlebnisse des Schneiders in indirekter Rede und damit auf eine Distanz signalisierende Weise erzählt, so läßt er in den exemplarischen Schilderungen der ,Schelmenzucht' den Schneider sich in direkter Rede an sein Publikum wenden. Die dadurch bewirkte Unmittelbarkeit erleichtert die Solidarisierung der Rezipienten mit den Beraubten und erhöht das provokative Potential des Textes.

Auf die Steuerung der Rezeption zielt schließlich auch die konsequent ironische Struktur des Textes. Die bereits konstatierte Durchbrechung normierter Wort-Sinn-Bezüge in den Reden des Schneiders und des Baßgeigers ist nicht nur als Kunstgriff der jeweils sprechenden Figur zu verstehen, sie erweist sich als vom Erzähler inszenierte Strategie, die der Schaffung einer durch Konsens konstituierten Kommunikationsgemeinschaft zwischen Kalendermann und Leserschaft dient. Die Umwertung vertrauter sprachlicher Termini, die Verwendung eines gegensätzlichen Ausdrucks an Stelle des eigentlich gemeinten und adäquaten als Merkmal ironischer Schreibart bietet die Möglichkeit, Erzähler und Leser zu Komplizen zu machen. Solange die Adressaten der Schelmenzucht an den durch sprachliche Konventionen vorgegebenen Relationen zwischen signifiant und signifié und den damit verknüpften normativen Setzungen festhalten, sind sie in der Lage, die Ironiesignale zu deuten, und die durch die Textstruktur bewirkte Entlarvung der ,Schelmenzucht" nachzuvollziehen.

${ }^{45} \mathrm{SW} 23, \mathrm{~S} .197$.

46 Vgl. SW 23, S. 201: „einige meinten, einen solchen König, der solche Weisungen gebe, sollte man bei den Beinen aufhängen." 
Die indirekte Didaxe in Die Schelmenzucht dient letztlich der gleichen Intention wie die explizite Ermahnung, die in den Predigten sowie in einzelnen Kalenderbeiträgen, die sich mit theologischen und ethischen Fragen befassen, dominiert. Durch eine Textorganisation, die durch Fiktionalitätssignale die Glaubwürdigkeit der Darstellung des Schneiders unterminiert, die das Kalenderpublikum daraufhin als potentiell betroffenes anspricht, dieser Betroffenheit durch Figuren Gestalt verleiht und damit Identifikationsmuster anbietet sowie mittels Ironie das zu Verwerfende demaskiert und die Rezipienten zur Solidarisierung mit der vom Erzähler vertretenen Haltung motiviert, versucht Gotthelf, seiner Botschaft Gehör zu verschaffen. Die satirische Denunziation der ,Schelmenzucht' hat zwar zunächst die auf eine Humanisierung der Justiz gerichteten Bestrebungen der Berner Regierung im Visier, sie dient jedoch darüber hinaus einer nicht nur sittlichen, sondern auch religiösen Bildung der Leser, zielt auf die ,wahre, vernünftige Aufklärung, die dem ächten Christen geziemt ${ }^{447}$ und die das Individuum befähigen soll, klar zwischen Gut und Böse, Recht und Unrecht zu unterscheiden. Den Maßstab für die Beurteilung irdischer Erfahrung bilden dabei jene religiös fundierten Ordnungsprinzipien, die, nach Auffassung des Emmentaler Pfarrers, transzendente und immanente Welt gleichermaßen durchwirken.

3.

Der notwendigerweise selektive Blick auf die durch Jeremias Gotthelf betreuten Jahrgänge des Neuen Berner-Kalenders hat es ermöglicht, die narrative Spezifik der Publikation präziser zu erfassen. Was die Tradition an Darstellungsmustern bereithält, wird auf durchaus eigenständige Weise umgesetzt in ein Kalenderkonzept, das sich gerade hinsichtlich seiner kommunikativen Struktur von vergleichbaren Publikationen abhebt. Als oberstes Ziel seiner Bestrebungen als Kalendermacher hatte Gotthelf in seinen programmatischen Äußerungen die „Bekehrung ${ }^{48}$ seiner Leser zu einem gottgefälligen Leben bestimmt. Erreicht werden soll dies nicht in erster Linie durch geistliche Unterweisung, sondern durch die bewußte Instrumentalisierung der durch die Publikationsform vorgegebenen Möglichkeiten. Die nicht unproblematische Rolle, die Gotthelf dem Kalenderschreiber zuweist, ist diejenige eines Autors, der einerseits als moralische Instanz unbedingte Aufmerksamkeit beanspruchen darf, der jedoch andererseits seine Leser als Partner eines Dialogs mit literarischen Mitteln ernst zu nehmen hat. Die Analyse der Schelmenzucht hat nun allerdings gezeigt, daß poetische Intention und publi-

47 EB 11, S. 36.

${ }^{48} \mathrm{Vgl}$. die bereits zitierte programmatische Äußerung in SW 23, S. 13. 
zistische Realisation keinesfalls kongruent sind, daß Gotthelf die Aporie einer zugleich monologischen und dialogischen Kommunikation in der literarischen Praxis nicht aufzulösen vermochte. In der Mehrzahl seiner Beiträge zum Neuen Berner-Kalender bedient sich der Emmentaler Theologe einer Aussagestruktur, die auf eindeutige Botschaften ausgerichtet ist und den Rezipienten wenig Raum läßt für autonome Meinungsbildung. Die kunstvolle, auf eine Vielzahl rhetorischer und poetischer Mittel rekurrierende Textgestaltung in Die Schelmenzucht zielt auf eine Identifikation des Publikums mit dem Kalendermacher und den ihm zugeordneten Figuren. Die im Text vertretenen Positionen werden dabei nicht im Rahmen einer expliziten Belehrung argumentativ entfaltet, sondern durch die Konfrontation mit einer Auffassung, deren Unzulänglichkeit augenfällig erscheint, bestätigt. Eine derartige Verständigung zwischen Kalendermann und Leserschaft setzt allerdings die Kompatibilität der Werthaltungen von Autor und Rezipienten voraus. Die Identifikation mit den von der Erzählerfigur repräsentierten Anliegen wird nur dort gelingen, wo der Eigentumsbegriff und die Rechtsauffassung des Kalenderpublikums mit den der Erzählung zu entnehmenden Postulaten übereinstimmen. Was der Text mit seinem konsequent ironischen Duktus versucht, ist die Schaffung einer Komplizenschaft zwischen Kalendermann und Kalenderleser, die Konstitution einer Kommunikationsgemeinschaft, die auf Konsens basiert. Dieser Konsens erscheint nicht als Herbeizuführendes, sondern als bereits Vorhandenes, er ist das Ergebnis eines in den Rezipienten angelegten Denkmusters, das einer Aktualisierung bedarf, die der Text bewerkstelligt. Die hier beschriebene Textstrategie offenbart, in welchem Maße es Gotthelf darum geht, ein letztlich religiös verortetes Normensystem auf unmißverständliche Weise zu vermitteln. Daß er als Kalenderautor gleichermaßen als Theologe und Satiriker in Erscheinung tritt, daß er die idealtypische Darstellung christlicher Normen in den Paränesen durch die ironische Entlarvung unchristlichen Denkens und Handelns in den satirischen Beiträgen ergänzt, hat nicht nur die Attraktivität des Neuen Berner-Kalenders erhöht; der komplementäre Gebrauch unterschiedlicher Schreibarten dient auch dazu, auf besonders einprägsame Weise Bedeutung zu generieren.

Inwiefern nun hat der Neue Berner-Kalender die vom Autor definierten publizistischen Zielsetzungen eingelöst? Gotthelfs Medium entspricht der Intention seines Autors insofern, als die Botschaften, die er seinen Lesern vermittelt, den Anspruch auf axiomatische Gültigkeit erheben; die an anderer Stelle geforderte Institutionalisierung eines Dialogs zwischen dem Schriftsteller und seinem Publikum dürfte angesichts der den Kalenderbeiträgen eignenden Kommunikationsstruktur nur in begrenztem Maße gelungen sein. Zwar läßt der Berner Dichter den Kalendermann als Figur auftreten, die derjenigen Bevölkerungsschicht angehört, aus der auch die potentiellen Käufer des Neuen Berner-Kalenders stammten, und versucht, durch Fortset- 
zungsgeschichten und wiederkehrende Rubriken seine Leser zu einer Gemeinschaft von Eingeweihten werden zu lassen und sie so an das von ihm veröffentlichte Periodikum zu binden. Die Resonanz, auf die die Kalenderjahrgänge 1840 bis 1845 stießen, $^{49}$ kann jedoch als Indiz dafür gedeutet werden, daß es Gotthelf nicht geglückt ist, die Kommunikation in Gang zu setzen, die er als Bedingung erfolgreicher publizistischer Tätigkeit definiert hatte. Der vergleichsweise mäßige Erfolg des Newen Berner-Kalenders ${ }^{50}$ dürfte sehr wesentlich mit den hier beschriebenen für Gotthelfs Publikation konstitutiven narrativen Strategien zusammenhängen, wie ein kurzer Blick auf die ungleich beliebteren Kalender von Gottlieb Jakob Kuhn und Johann Peter Hebel zeigt: $Z$ war spart auch Kuhn in seinem Hinkenden Boten nicht mit Kritik am politischen Radikalismus. Die Mäßigung und der Humor, mit denen er zeitgenössische Lebenswirklichkeit darlegt und reflektiert, dürften allerdings auch diejenigen Leser, die die konservative Haltung des Pfarrers von Burgdorf nicht teilten, angesprochen haben. In noch stärkerem Maße als der Hinkende Bote bemüht sich Der Rbeinländische Hausfreund darum, mit einem breiten und heterogenen Publikum ins Gespräch zu kommen. Durch eine Textgestaltung, die auf subtile Weise die Rezeption des Erzählten steuert, wird in Hebels Erzählungen ein intellektueller Prozeß in Gang gesetzt, der auf eine Erkenntnis zuläuft, die jenseits eindeutiger Botschaften liegt. Hebels Kalendergeschichten ermöglichen verschiedene Interpretationen, sie vermitteln nicht „fixierte Bedeutungen, sondern eröffnen einen Spielraum möglicher Bedeutungen, der zur Sinnkonstitution durch den Leser auffordert. ${ }^{51}$ Durch kluge Berücksichtigung der stofflichen und formalen Lesebedürfnisse der Rezipienten, und durch die Fokussierung des Erzählens nicht auf die durch die narratio angebotene potentielle Bedeutung, sondern auf die Bedeutung konstituierende Textaneignung, kann der Herausgeber des Rheinländischen Hausfreunds auch in denjenigen Kreisen mit geneigten Lesern rechnen, die nicht zu seinen unmittelbaren Adressaten zählen. ${ }^{52}$ Ganz anders verhält es sich mit Gotthelfs Kalender. Die Entscheidung

19 Zur Rezeption des Neuen Berner-Kalenders vgl. Hunziker: Der Neue Berner-Kalender, S. 125-144.

${ }^{50}$ Gotthelfs Neuen Berner.Kalender als verlegerischen Mißerfolg zu bezeichnen, wäre übertrieben. Die für das Jahr 1843 ausgewiesene Auflage von 12.000 Exemplaren, von denen allesdings nur knapp 11.000 abgesetzt werden konnten (vgl. Hunziker: Der Neue Berner-Kalender, S. 124), belegt, daß die Publikation durchaus Verbreitung gefunden hat. Gotthelf selber äuBert sich in einem Brief vom 27.10.1840 an Joseph Burkhalter durchaus optimistisch zu seinem Kalender, betont der „Erfolg [sei] kein ungünstiger, und außer Distelis [werde] wohl kaum einer so viel gelesen als dieser" (EB 5, S. 89).

51 Rusterholz: Faktoren der Sinnkonstitution, S. 111.

52 Vgl. Hebels Brief an das badische Innenministerium vom 17.9.1811, in dem er den Erfolg seines Kalenders in „alle [n] Provinzen des deurschen Sprachgebiets" und selbst bei den nersten und berühmtesten Männern der Nationen“ erwähnt (vgl. Rohner: Kommentarband zum Rbeinländischen Hausfreund, S. 87). 
zugunsten einer narrativen Strategie, die Konsens zwischen Autor und Leser nicht argumentativ herbeiführt, sondern voraussetzt, begrenzt die Reichweite der Publikation. Abnehmer fand der Neue Berner-Kalender wohl primär unter denjenigen Bevölkerungsgruppen, die die politische und theologische Auffassung des Verfassers teilten. Daß diese Auffassung in den 1840er Jahren nicht mehrheirsfähig war, läßt das bemerkenswerte Echo, auf das der seit 1839 erscheinende Schweizerische Bilderkalender stieß, vermuten. ${ }^{33}$ Der Erfolg des vom Zeichner Martin Disteli herausgegebenen Kalenders kann nicht nur mit der im Titel dokumentierten überregionalen Ausrichtung und der Qualität der den Texten beigegebenen Illustrationen begründet werden, er hängt auch zusammen mit der politischen Ausrichtung der Publikation, die dezidiert radikale Positionen vertrat. Wie der Neue Berner-Kalender spart auch der Schweizerische Bilderkalender nicht mit Kritik insbesondere am Verhalten der konservativen politischen und kirchlichen Eliten. Anders als Gotthelf jedoch, dessen eigenwillige Unzeitgemäßheit Gottfried Keller in seiner Rezension zu den Uli-Romanen kritisch vermerkt, ${ }^{54}$ konnte Disteli, dort, wo er sich in satirischer Absicht an sein Publikum wandte, mit der Zustimmung breiterer Kreise rechnen. Die durch die Forschung angebotenen Erklärungmuster für den begrenzten Erfolg des Neuen Berner-Kalenders - daß Gotthelf als Verfasser und Herausgeber des Neuen Berner-Kalenders die Lesegewohnheiten seines Publikums nur ungenügend berücksichtigt, daß er durch eine Fülle von Allusionen seine Leser nicht selten überfordert, daß er durch die Schärfe, mit der er zu politischen Entwicklungen Stellung nahm, sich zahlreiche Feinde geschaffen habe ${ }^{55}$ - sind nicht von der Hand zu weisen. Nicht weniger entscheidend ist jedoch, daß der Neue Berner-Kalender sich zur Vermittlung restaurativen Gedankenguts einer narrativen Strategie bedient, die auf einen Konsens zwischen Autor und Publikum ausgerichtet ist, für den im zunehmend von liberalen Ideen geprägten Rezipientenkreis populärer Literatur die Voraussetzungen immer weniger gegeben waren. Die für Gotthelfs Kalender konstitutive Mischung aus formaler sowie motivischer Innovation und politischem Konservatismus dürfte im Gegenteil die Leser eher irritiert als für die Anliegen des Verfassers eingenommen haben.

Gotthelfs Engagement für den Neuen Berner-Kalender gründet wesentlich in der Einsicht, daß mit einem breitere Publikumsschichten erschließenden Periodikum ungleich größere Wirkung zu erzielen war als mit Romanen und

${ }^{53}$ Der Schweizerische Bildkalender erreichte in kürzester Zeit eine Auflage von rund 26.000 Exemplaren (vgl. Rohner: Kalendergeschichte und Kalender, S. 329-393).

54 Vgl. Keller: Sämtliche Werke, Bd. 22, S. 58-59: Bitzius sagt in einer Vorrede: man werde ihm wenigstens nicht ein gedankenloses und feiles Segeln mit herrschenden Winden vorwerfen können. Das ist allerdings sehr wahr; er verfält aber in das andere Extrem und sucht mit dem größten Eigensinn gegen den Strom zu schwimmen, und das ist für einen Volksschriftsteller auch nicht klug und weise."

${ }^{55}$ Vgl. beispielsweise Hunziker: Der Neue Berner-Kalender, S. 146-150. 
Erzählungen, die nur von einer vergleichsweise begrenzten Zahl von Lesern gekauft und gelesen wurden. Ob die Publikation die erhoffte Wirkung gezeitigt hat, bleibt fraglich, obwohl Gotthelf, wie die programmatischen Äußerungen in seiner Korrespondenz belegen, die spezifischen Möglichkeiten des Mediums im Hinblick auf sein Kalenderkonzept reflektiert und, zumindest partiell, fruchtbar gemacht hat. $Z$ war sind die Bezüge zwischen Kalender und poetischem Werk eng. Sowohl in thematischer als auch in formaler Hinsicht ließen sich Übereinstimmungen belegen. Der Kalender kann, wie das Beispiel der Kritik an der Quacksalberei veranschaulicht, auf komplementäre Weise an sich im Romanwerk manifestierenden Auseinandersetzungen mit politischen, ökonomischen und sozialen Problembereichen partizipieren; er dient zugleich als poetisches Laboratorium, in dem Gotthelf Motive und narrative Verfahrensweisen erprobt, die er in der in den 1840er Jahren erschienenen Erzählprosa einsetzt. ${ }^{56} \mathrm{Daß}$ die Kalendertätigkeit des Berner Dichters nicht einen ,Seitentrieb" darstellt, wie dies die ältere Forschung behauptete, ${ }^{57}$ sondern in dessen Schaffen eine zentrale Stelle einnimmt, daß zwischen dem Neuen Berner-Kalender und den zeitgleich entstandenen Romanen und Erzählungen ein bisweilen osmotisches Verhältnis besteht, bedeutet jedoch nicht, daß Gotthelf das besondere kommunikative Potential des Volkskalenders nicht erkannt hätte. In Kenntnis der für das Medium kennzeichnenden Interaktionsmuster hat er eine Neubestimmung des Verhältnisses zwischen Autor und Publikum versucht, und dabei auf eine Erzählstrategie gesetzt, die um größtmögliche Evidenz bemüht ist. Die Beiträge zum Neuen Berner-Kalender mögen nicht immer die ästhetische Komplexität der frühen Romane und Erzählungen besitzen, sie belegen jedoch auf eindrückliche Weise, wie eigenwillig Gotthelf tradierte literarische Muster neuen Funktionsmöglichkeiten dienstbar gemacht hat.

56 Vgl. dazu Utz: Gezügeltes Erzählen. Die beiden Fassungen von Gotthelfs Mordiofubrmann. Der bekebrte Mordiofubrmann ist nicht die einzige Kalendergeschichte, die Gotthelf in einer mehr oder weniger überarbeiteten Fassung noch einmal veröffentlicht hat. Zu den später in den Geschichten und Sagen aus der Schweiz und den Erzablungen und Bildern asus dem Volksleben der Schweiz wiederaufgelegten Kalenderbeiträgen vgl. SW 24, S. 362-388.

57 Vgl. Itten: Gotthelf als Kalenderschreiber, S. 5. 